\title{
Structural, kinematic and kinetostatic analysis of a manure spreader equipped by a mobile platform with articulated elements
}

\author{
Lucretia Popa ${ }^{1, *}$, Stefan-Iulian Moise ${ }^{2}$, Victor Moise ${ }^{3}$, \\ Vasilica Stefan $^{1}$ and Ioan Ganea-Christu ${ }^{1}$, \\ ${ }^{1}$ INMA Bucharest, 6 Ion Ionescu de la Brad Blvd., Bucharest, Romania \\ ${ }^{2}$ Etudes et Productions Schlumberger, Paris, France \\ ${ }^{3}$ University POLITEHNICA of Bucharest, Romania
}

\begin{abstract}
Organic fertilization involves working with machines by a special construction, suitable for the material to be spread, machines that have, in most cases, chain conveyors with scrapers attached to the chains. In some cases, the conveyor chain may break, due to the jams, and the need for manual unloading of the bunker (bucket) appears, which requires a great physical effort, the unloading being made by the operator, using a shovel. The construction of the platform using mobile, articulated elements would greatly facilitate the unloading at the bottom of the bunker and would eliminate the physical effort of the operator. This article presents the structural, kinematic and kinetostatic analysis of a mechanism designed for actuating a mobile platform, which is part of a manure spreader, composed of articulated elements driven by hydraulic cylinders.

For the kinematic analysis of the mobile element platform's mechanism, calculation procedures written in the Matlab syntax are used.

After the kinematic analysis of the mechanism, the kinetostatic analysis is performed, i.e. the determination of the reactions from the kinematic couplings, as well as the hydraulic force from the hydraulic cylinder. The results can be used for dimensional verification of the mechanism components, thus contributing to the optimization of the mechanism.
\end{abstract}

\section{Introduction}

The technological trailers that carry out the transport and distribution of bulk fertilizers (chemical fertilizers, compost etc.) have a special construction.

Over time, there have been many researches that aimed to improve the construction in order to improve the quality of the work, or for the ease of carrying out the work or the machine maintenance. [1-6].

The technological trailers, have usually a monocoque construction, and can perform only the rear discharge. Most of the time, the material is distributed with the help of a spreading device with different construction, but usually, the material is transported inside

* Corresponding author: Lucreţia Popa, lucretia popa@yahoo.com 
the trailer, to the spreading device, with the help of a conveyor with scrapers arranged on the chain.

The drag chain is on the bottom of the bin to pull the load of manure to the rear where it is shredded by a pair of beaters and spread on the ground. But there are many situations when the chain is broken, because of the manure which contains different objects like wires, stones and in this case, it is difficult to discharge the machine using un unloading equipment like a front loader mounted on the tractor or manually, using shovels.

To offer to the operator the possibility to discharge the machine when the chains are broken, this article studies the possibility to design a bin with many platforms, jointed and driven by a motor group (motor dyad), by means of parallelogram mechanisms.

This idea was transposed into a patent application submitted to the Romanian State Office for Inventions and Trademarks-OSIM. [7].

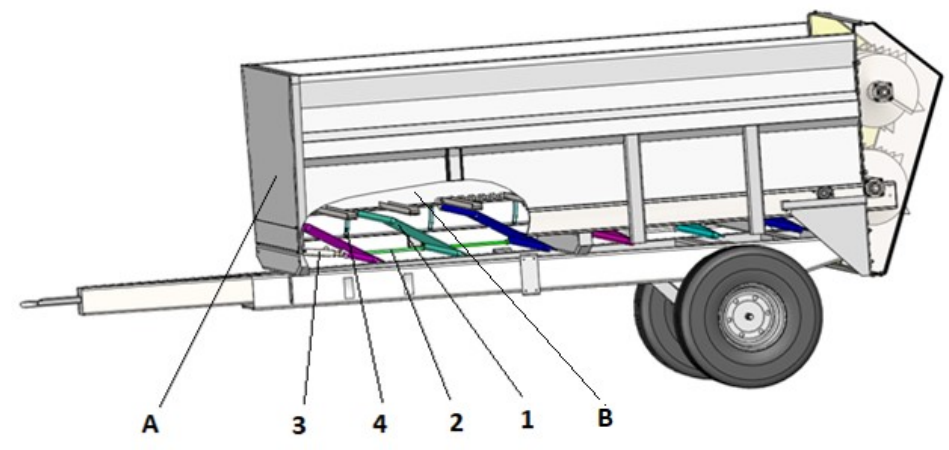

a)

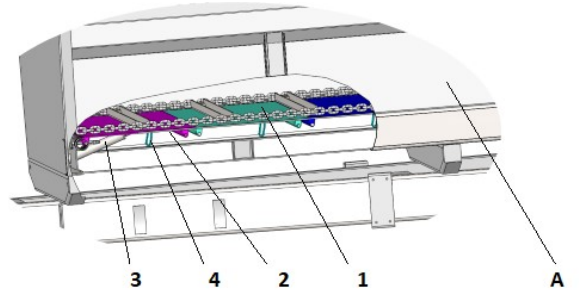

b)

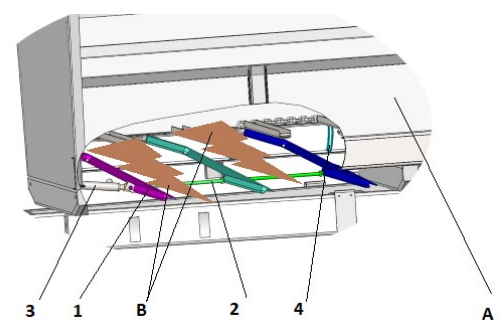

c)

Fig. 1. Manure spreading machine with joint elements [7]

a) left side perspective view; b) detail in the horizontal position; c) open platform

The construction relates to a platform with articulated movable elements, which is part of the trailer bin A, in order to improve the process of unloading the material in case of damage.

The platform consists of a number of movable elements 1 , joint, the system 2 of bars that connects the movable elements and which ensures their inclination, the hydraulic cylinders 3 , the lateral guides 4 which ensure the steering of the movable elements and the limitation of the maximum angle of inclination of the elements movable during the process of unloading material B remaining in the trailer.

In this article we study the mechanism characteristics, to obtain information for development of this idea and to apply it in the real design and manufacturing. In this paper, structural, kinematic and kinetostatic analysis of the mechanism acting the mobile platforms is performed. The determination of the reactions of the kinematic couplings is necessary for the dimensioning calculation. For the actuation of the entire mechanism, the 
size of the hydraulic cylinder motor force must be determined, which is made using the kinetostatic method and the virtual powers method.

\section{Material and method}

Figure 2 shows the kinematic diagram of the mechanism designed for acting the jointed movable elements, which are part of the platform.

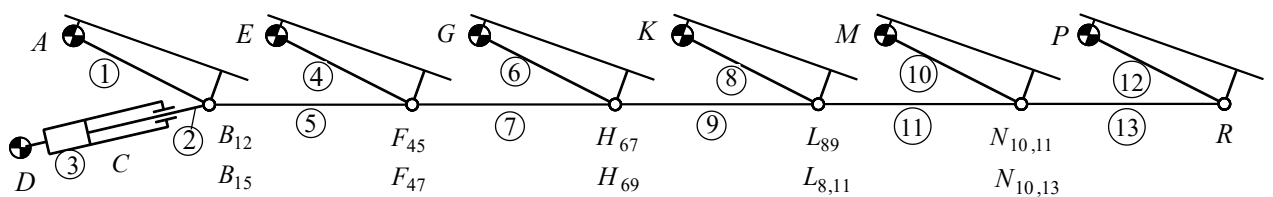

Fig. 2. Kinematic scheme of the mobile platforms driving mechanism

In order to determine the kinematic parameters of the mechanism's elements, it is necessary to know the structural groups in its composition, which means, the structural analysis of the lifting and folding mechanism of the platform jointed elements must be performed.

For the structural, kinematic and kinetostatic analysis of the mechanism, the kinematic diagram of the mechanism is known, also the dimensions of the kinematic elements and the positions of the couplings adjacent to the base, the initial position of the mechanism, the kinematic parameters in the C-motor coupling, the masses and the mechanical moments of inertia of the elements, as well as the technological forces on each individual platform.

\section{Results}

\subsection{Structural analysis of the mechanism}

The structural analysis of the mechanism consists in establishing kinematic couplings, determining the mobile elements, establishing the degree of mobility, drawing up the structural scheme, establishing the structural structures of the mechanism, constructing the multipolar scheme and establishing the structural relationship [10-15].

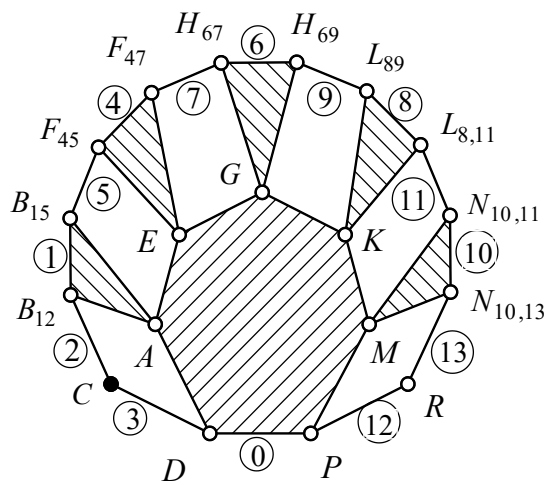

Fig. 3. Structural scheme of the mechanism

Observing the relative movements between the elements of the mechanism, (Figure 3), it is found that it has the following inferior couplings: $A(0 R 1), B_{12}(1 R 2), C(2 T 3), D(3 R 0)$, 
$B_{15}(1 R 5), E(4 R 0), F(4 R 5), F(4 R 7), G(6 R 0), H(6 R 7), H(6 R 9), K(8 R 0), L(8 R 9), L(8 R 11)$, $M(10 R 0), N(10 R 11), N(10 R 13), P(12 R 0)$ and $P(12 R 13)$. The number of superior couplings is zero. The mobile elements of the mechanism are: $1\left(A, B_{12}, B_{14}\right), 2\left(B_{12}, C\right), 3(C, D), 4(E$, $\left.F_{45}\right), 5\left(B_{15}, F_{45}\right), 6\left(G, H_{67}\right), 7\left(F_{47}, H_{67}\right), 8\left(K, L_{89}\right), 9\left(H_{69}, L_{89}\right), 10\left(M, N_{10,11}\right), 11\left(L_{8,9}, N_{10,11}\right)$, $12(P, R)$ and $13\left(B_{10,13, R)}\right.$.

Taking into account the number of moving elements and the number of kinematic couplings, the degree of mobility of the mechanism results [10-15], namely $\mathrm{M}=1$, meaning that only one independent parameter is required for the elements of the mechanism to have unique determined movements. Figure 3 shows the structural diagram of the mechanism.

Figure 4 presents the multipolar scheme and its structural relationship.

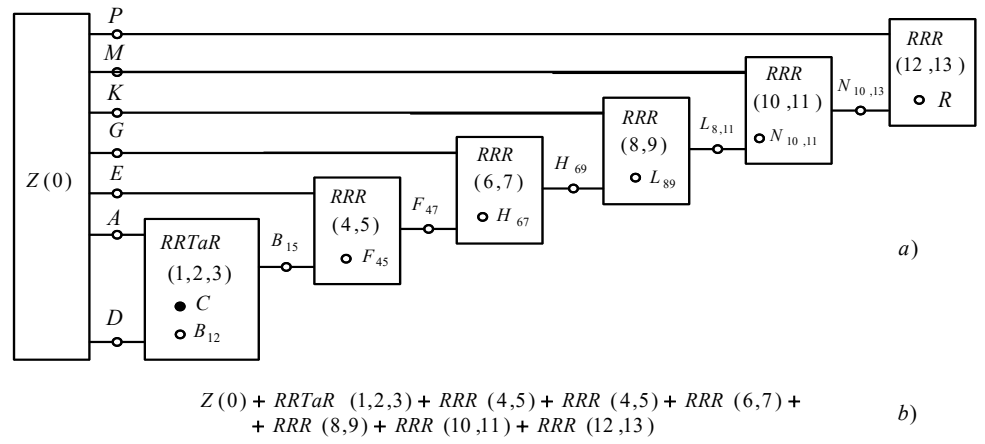

Fig. 4. Multipolar scheme of the mechanism and structural relationship

\subsection{Kinematic analysis of the mechanism}

Figures 2, 3 and 4 show that the mechanism consists of the base $Z(0)$ and the motor dyad $R R T a R(1,2,3)$ and five passive dyads of aspect 1 (RRR dyads).

Figure 5 shows the kinematic diagram of the mechanism, highlighting the kinematic parameters of position.

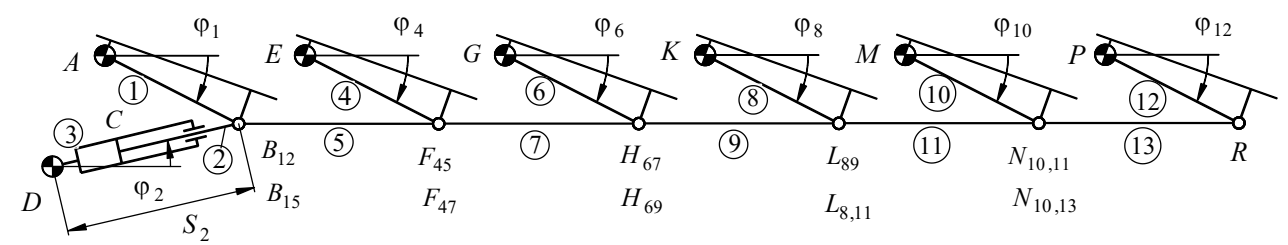

Fig. 5. The kinematic scheme of the mechanism, highlighting the position of parameters

For the kinematic analysis of the mechanism, calculation procedures written by the authors in the Matlab syntax are used. Figures 6 and 7 show diagrams of variation of angular velocities and accelerations of the motor dyad elements. 


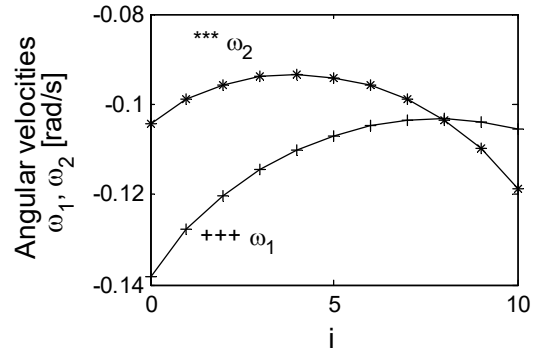

Fig. 6. Angular velocity diagrams $\omega_{1}$ and $\omega_{2}$

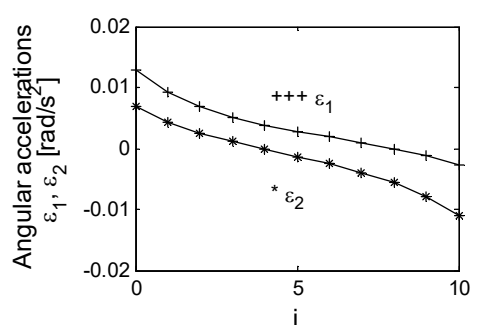

Fig. 7. Angular acceleration diagrams

$$
\varepsilon_{1} \text { and } \varepsilon_{2}
$$

Note: $i$ represents the calculation position at a given time. The distance between two calculation positions (on the $x$-axis) is an increment. This is equal to the stroke of the piston / (n-1), where $n$ is the number of calculation positions (the starting position is also considered).

\subsection{Kinetostatic analysis of the mechanism}

Kinetostatic analysis of the mechanism is done in the opposite direction of the kinematic analysis, that is, starting with the $R R R(12,13)$ dyad and ending with the RRTaR $(1,2,3$,$) motor group.$

For the kinetostatic analysis, the technological forces, the forces of inertia and the forces of weight of the elements are taken into account.

Figures 8-13 show the diagrams of the forces and moments that act on the component elements of the dyads and the kinetostatic diagrams.

Table 1

\begin{tabular}{|c|c|}
\hline $\begin{array}{l}\text { Highlighting the forces and } \\
\text { moments acting on the dyads' elements }\end{array}$ & Dyads kinetostatic diagrams \\
\hline$\left.\bar{F}_{i 13}\right|_{G_{13}} ^{M_{i 13}}$ & $N \underbrace{\bar{R}_{0,12 Y}}_{\bar{R}_{11,13 X}}$ \\
\hline a) & b) \\
\hline \multicolumn{2}{|c|}{ Fig. 8. $R R R(12,13)$ dyad } \\
\hline (11) & $L{\overline{\bar{R}_{9,11 X}}}_{G_{11}}^{\bar{R}_{0,10 Y}}$ \\
\hline ig. 9. $R$ & $0,11)$ dyad \\
\hline
\end{tabular}




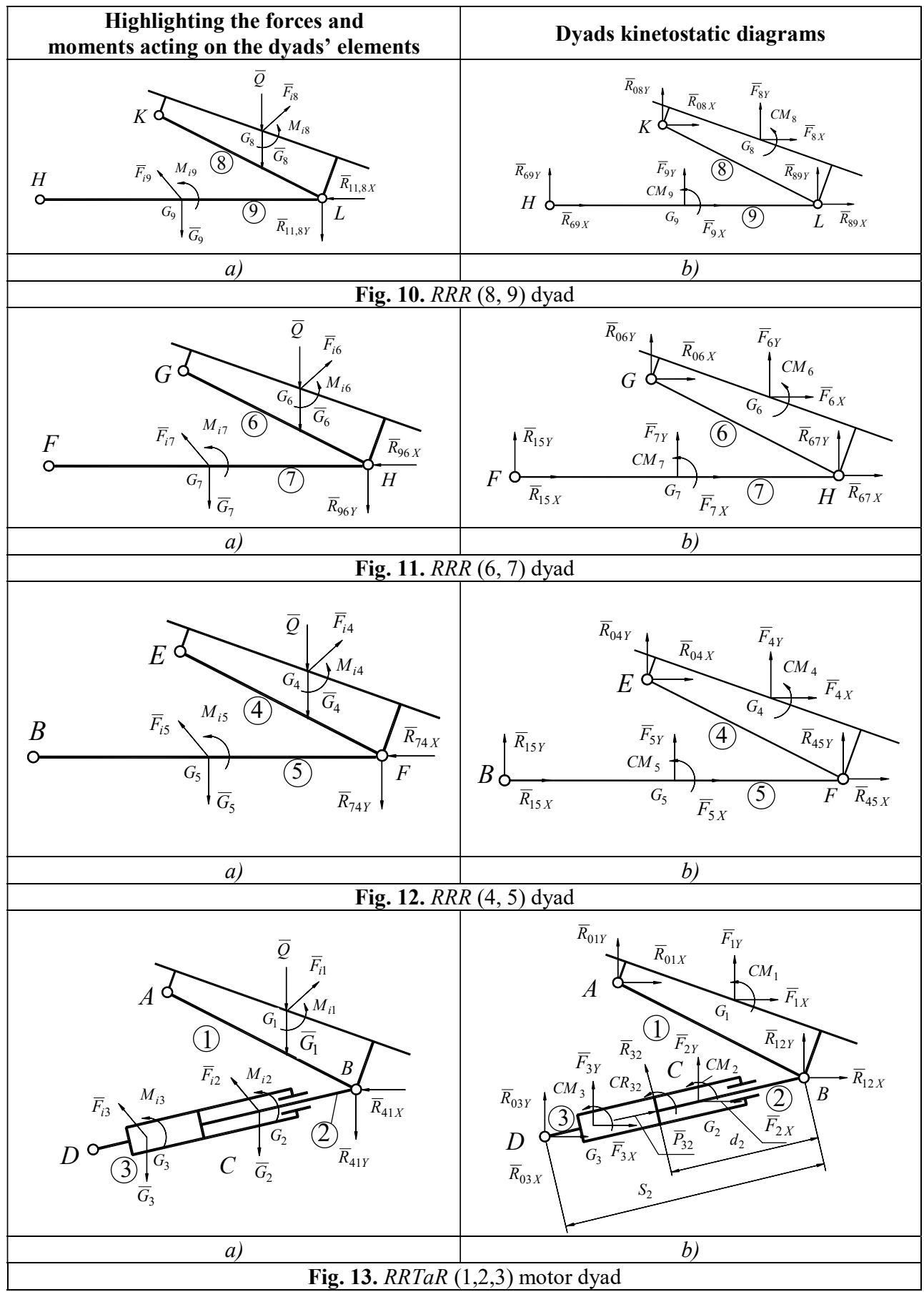

Figures 14, 15, 16 and 17 show the hodographs of the reaction forces of the kinematic couplings $A, B, D$ and $E$, and in figure 18 the diagram of the motor force variation in the hydraulic cylinder is presented. 


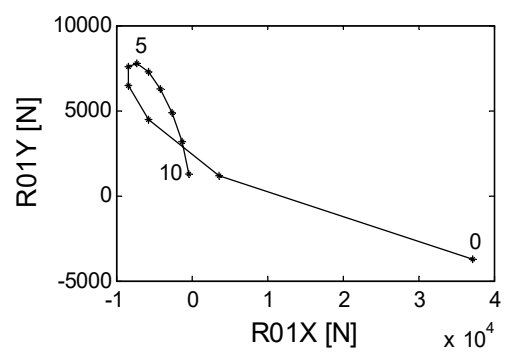

Fig. 14. Hodograph of the reactions of the coupling $A$

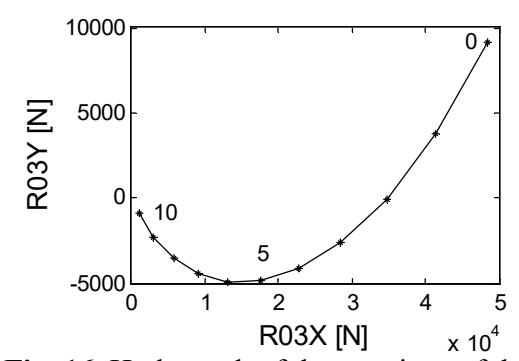

Fig. 16. Hodograph of the reactions of the coupling $D$

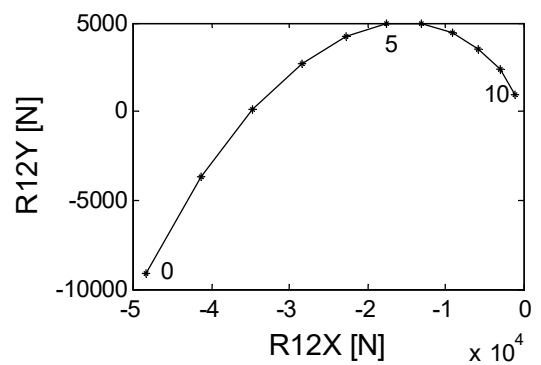

Fig. 15. Hodograph of the coupling $B$

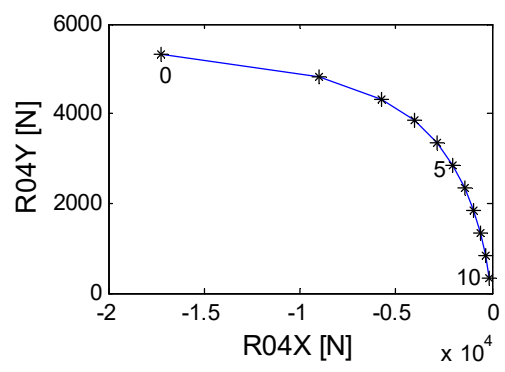

Fig. 17. Hodograph of the coupling E

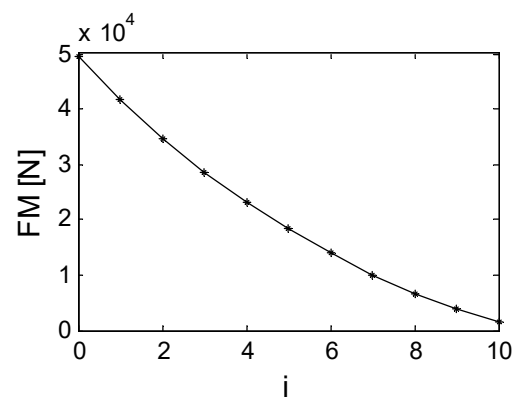

Fig. 18. Chart driving force in the coupling $C$

Table 2 presents the values of the driving force in the hydraulic cylinder, for a cycle of operation, values calculated by the kinetostatic method (FE_CINET) as well as by the virtual powers method (FE_PVIR).

\section{Table 2}

pos FE_CINET FE_PVIR

\begin{tabular}{|c|c|c|}
\hline & 49328.67 & 49328.67 \\
\hline & 41576.80 & 41576.94 \\
\hline 2 & 34739.41 & 34739.41 \\
\hline 3 & 28648.28 & 28648.28 \\
\hline 4 & 23199.73 & 23199.73 \\
\hline 5 & 18325.45 & 18325.45 \\
\hline & 13978.50 & 13978.50 \\
\hline & 10126.10 & 10126.10 \\
\hline & 6745.94 & 6745.94 \\
\hline & 3824.86 & 3824.86 \\
\hline & 1359.63 & 1359.63 \\
\hline
\end{tabular}


Analysing the data in table 2, it is observed that the same values were obtained by two methods of calculation, which means that all the calculations were correct and the data obtained can be used to dimension the mechanism that acts the platform with articulated movable elements.

\section{Conclusions}

The structural and kinematic analysis of the mechanism provided useful information about the functionality of the system, showing its correct functioning, if it has the degrees of freedom required for a movement without blockages and dysfunctions, the conclusion being that the mechanism was well designed in terms of structure.

The kinetostatic analysis made by the simulation enables in the future an optimal dimensioning of the elements that form the mechanism of the platform with multiple articulated mobile elements. An over dimensioning of the system can increase the price of the machine or, a subdimension causes damages of the system, and unwanted unloading of the material transported. The kinematic analysis realized in this paper is necessary for the dynamic analysis, which will be performed in the following research.

This work was supported by a grant of the Romanian Ministry of Education and Research, through Programme 1 - Development of the National Research-Development System, subprogramme 1.2 - Institutional performance - Projects for financing excellence in RDI, contract no. 16PFE.

\section{References}

1. P. Cârdei, V. Stefan, L. Popa, R. Ciuperca, Statistical models for the working process carried out by the organic fertilizer spreading machine, INMATEH Agricultural Engineering, 58/2, 121-128, (2018)

2. L. Popa, L. Dudici, A. Nedelcu, A.C. Cristescu, D.M. Dumitru, Kinetostatic analysis of ratchet mechanism that acts on conveyor of manure spreading machine, Engineering for Rural Development-ERDev2018, 57-63, DOI: 10.22616/ERDev2018.17, (2018)

3. K. Sengottaian, N. Mohanrajhu, S. Palani, C. Jayabalan, Design of Manure Spreader Machine for agricultural form field, International Journal of Innovative Technology and Exploring Engineering-IJITEE, 9/1, 4280-4284, DOI: 10.35940/ijitee.A4877.119119, (2019)

4. V. Ştefan, P. Cârdei, L. Popa, L. David, R. Ciupercă, Influence of the manure spreading machines' working parameters on the qualitative performances of the fertilization process, INMATEH Agricultural Engineering, 58/2, 115-120, (2018)

5. V. Stefan, P. Cârdei, V.N. Vlăduţ, L. Popa, R. Ciuperca, N. Ungureanu, Mathematical model for particle motion applied on a manure spreading apparatus used in environmentally friendly technology, EEMJ, 17/1, 217-227, (2018)

6. V. Ştefan, R. Sfîru, L. Popa, Experimental results on the solid organic fertilizer machine $M G$ 5, TE-RE-RD, (2019)

7. L. Popa, A. Nedelcu, V. Ştefan, A.C. Cristescu, Platform with mobile elements for trailers Patent request no. A/00824/22.10.2018, OSIM, (2018)

8. I.I. Artobolevski, Théorie des Mécanismes et des Machines (Theory of Mechanisms and Machines). 453 p., Mir Publishing House, (1977)

9. B. Demidovich, A.I. Maron, Elements of numerical calculation (Éléments de calcul numérique), $2^{\text {nd }}$ edition, 717 p., Mir Publishing House, (1987)

10. C. Duca, Fl. Buium et al. Mechanisms, Gh. Asachi Publishing House, (2003) 
11. V.Moise, E. Maican, Şt.I. Moise, Numerical methods. MATLAB Applications (Metode numerice. Aplicații în MATLAB), 292 p., Bren Publishing House, (2016)

12. V. Moise, I. Simionescu, M. Ene, A. Rotaru, Analysis of plane mechanisms with hitch bars. MATLAB Applications (Analiza mecanismelor plane cu bare articulate. Aplicații in MATLAB), 324 p, Printech Publishing House, (2015)

13. Chr. Pelecudi. The precision of the mechanisms, Publishing House of the Academy of RSR, 398 p. (1975)

14. Chr. Pelecudi, D. Maroş, V. Merticaru, N. Pandre, I. Simionescu, Mechanisms (Mecanisme), 394 p., Didactic and Pedagogical Publishing House, (1985)

15. I. Simionescu, V. Moise, Mechanisms, Technical Publishing House, (1999) 